DRAFT VERSION NOVEMBER 5, 2018

Preprint typeset using LTTEX style emulateapj v. 08/22/09

\title{
RECOVERY FROM MAUNDER-LIKE GRAND MINIMA IN A BABCOCK-LEIGHTON SOLAR DYNAMO MODEL
}

\author{
BIDYA BINAY KARAK ${ }^{1,2,3}$ AND MARK MIESCH ${ }^{3}$ \\ ${ }^{1}$ Department of Physics, Indian Institute of Technology (Banaras Hindu University), Varanasi, India \\ ${ }^{2}$ Indian Institute of Astrophysics, Koramangala, Bangalore 560034, India \\ ${ }^{3}$ High Altitude Observatory, National Center for Atmospheric Research, 3080 Center Green Dr., Boulder, CO 80301, USA \\ (Dated: November 5, 2018) \\ Draft version November 5, 2018
}

\begin{abstract}
The Sun occasionally goes through Maunder-like extended grand minima when its magnetic activity drops considerably from the normal activity level for several decades. Many possible theories have been proposed to explain the origin of these minima. However, how the Sun managed to recover from such inactive phases every time is even more enigmatic. The Babcock-Leighton type dynamos, which are successful in explaining many features of the solar cycle remarkably well, are not expected to operate during grand minima due to the lack of a sufficient number of sunspots. In this Letter, we explore the question of how the Sun could recover from grand minima through the Babcock-Leighton dynamo. In our three-dimensional dynamo model, grand minima are produced spontaneously as a result of random variations in the tilt angle of emerging active regions. We find that the Babcock-Leighton process can still operate during grand minima with only a minimal number of sunspots and that the model can emerge from such phases without the need for an additional generation mechanism for the poloidal field. The essential ingredient in our model is a downward magnetic pumping which inhibits the diffusion of the magnetic flux across the solar surface.
\end{abstract}

Subject headings:

\section{INTRODUCTION}

The global magnetic field of Sun oscillates with polarity reversals every 11 years. This oscillation is well reflected by the number of sunspots observed on the solar surface and thus it is known as the sunspot cycle or the solar cycle. The solar cycle, however, is not regular. There was a time in the 17th century when the sunspot number, as well as other proxies of solar activity e.g., the auroral occurrence, went to an unexpectedly low value for about 70 years. This is the well-known Maunder minimum (Eddy 1976). From indirect terrestrial proxies of solar activity, we now know that this Maunder minimum is not unique and the Sun had many such events with different durations in the past (Beer et al. 1990; Solanki et al. 2004, Usoskin et al. 2007). The interesting fact is that every time the Sun manages to recover to the normal magnetic activity from these grand minima. In fact, we now know that the magnetic field during the Maunder minimum was oscillating, implying that the underlying process of magnetic field generation was still occurring during the grand minima (Beer et al. 1998. Miyahara et al. 2004).

It is believed that a magnetohydrodynamics dynamo process, operating in the solar convection zone $(\mathrm{CZ})$, is responsible for producing the solar magnetic cycle. At present, the Babcock-Leighton type flux transport dynamo model is a popular paradigm for the solar cycle because of its success in reproducing observations (Charbonneau 2010; Karak et al. 2014a). In this model, the decay and dispersal of tilted bipolar magnetic regions (BMRs) near the solar surface produce the poloidal field-the Babcock-Leighton process (Babcock 1961; Leighton 1964). The poloidal field is then transported to the bulk of the $\mathrm{CZ}$ through the turbulent diffusion and meridional circulation, where the winding of this field by differential rotation generates a toroidal field. This model is

Electronic address: karak.app@iitbhu.ac.in

Electronic address: miescn@ucar.eau constructed with an assumption that the toroidal flux near the base of the $\mathrm{CZ}(\mathrm{BCZ})$ produces BMRs at the surface. The observed tilt of BMRs relative to an east-west orientation is attributed to Coriolis force during the rise of the toroidal flux through the CZ (e.g., D'Silva \& Choudhuri 1993).

The BMR tilt is crucial in producing the poloidal field in this model (Dasi-Espuig et al. 2010). While in observations the tilt systematically increases with latitude-Joy's law, there is a considerable scatter around this systematic variation (Stenflo \& Kosovichev 2012; Wang et al. 2015, Arlt et al. 2016). This scatter in the tilt angle causes a variation in the polar field (Jiang et al. 2014, 2015, Hazra et al. 2017; Karak \& Miesch 2017, hereafter KM17). Based on this idea previous authors have included a random component in the Babcock-Leighton source of their flux transport dynamo models and have shown that this random component can diminish the poloidal source and trigger a grand minimum (Charbonneau et al. 2004; Choudhuri \& Karak 2009 2012; Karak \& Choudhuri 2013, Olemskoy \& Kitchatinov 2013; Hazra et al. 2014; Passos et al. 2014; Inceoglu et al. 2017). However, these models do not explicitly take into account realistic BMR properties such as tilt angle scatter, flux distribution, and cycle-dependent emergence rate. Furthermore, they do not fully explain how a Babcock-Leighton model can emerge from a grand minimum without some additional source of poloidal field such as a turbulent $\alpha$-effect. Recently, Lemerle \& Charbonneau (2017) (hereafter LC17) have developed a $2 \mathrm{D} \times 2 \mathrm{D}$ coupled surface flux transport and flux transport dynamo model in which the actual BMRs with observed properties have been included. They have demonstrated that explicit tilt angle fluctuations can indeed induce grand minima.

In a newly developed 3D dynamo model (KM17), we have included the tilt angle fluctuations explicitly and we have shown that the observed tilt scatter is capable of triggering grand minima events. When using the currently observed 
Gaussian fluctuations with $\sigma_{\delta}=15^{\circ}$, the occurrence of grand minima in the model is somewhat less frequent than that inferred from terrestrial proxies (Usoskin et al. 2007). However, a solar-like frequency is found when we double the scatter. Here we use the enhanced tilt-angle scatter of $30^{\circ}$ (double the observed value of $15^{\circ}$ ) in order to facilitate our analysis by producing more frequent grand minima. However, we emphasize that the mechanism we describe here for emerging from a grand minimum is not sensitive to the value used for the tilt-angle scatter; solar-like models emerge from grand minima in the same way. The essential emergence mechanism we describe (enabled by magnetic pumping) is also insensitive to other parameters of the model as well; one should be able to capture it even with a 2D model that does not have any explicit active regions.

Although previous studies demonstrate that tilt angle scatter can cause grand minima, they do not explain the recovery of the Sun from such phases. As BMRs are the only source for the generation of the poloidal field in the Babcock-Leighton type dynamos, the generation of poloidal field becomes negligible during grand minima due to a fewer number of BMRs. Thus the Babcock-Leighton dynamo may stop operating during grand minima and the Sun may not recover. Previous studies suggested that an additional poloidal source (e.g., convective $\alpha$ ) is needed in order for the Sun to recover from grand minima (Karak \& Choudhuri 2013; Hazra et al. 2014; Passos et al. 2014). Indeed, LC17 observed that when their model enters into an extended grand minimum of the weaker magnetic field, the generation of poloidal field stops due to lack of BMRs and the model never recovers from that quiescent phase. Their model recovers only when the magnetic field does not fall below a certain level.

\section{MODEL}

In this Letter, we explore the Babcock-Leighton dynamo mechanism during grand minima, focusing in particular on how the dynamo might recover from such episodes through the Babcock-Leighton process alone. To do so, we first produce grand minima. In our study, we build on our recent work, KM17 which is an updated version of the original model (Miesch \& Dikpati 2014; Miesch \& Teweldebirhan 2016). In this model, BMRs are produced near the surface based on the toroidal flux at the BCZ and most of the statistical properties of BMRs are based on solar observations. We refer the readers to Section 2 of KM17 for the details of this model. From KM17, we consider Runs B10-11 in which the diffusivity in the bulk of the $\mathrm{CZ}$ is in the order of $10^{12} \mathrm{~cm}^{2} \mathrm{~s}^{-1}$. The BMR flux distribution is fixed at the observed value and the time delay between two successive BMR emergences is obtained from the observed log-normal distribution which changes in response to the toroidal field at the base of the $\mathrm{CZ}$ to allow more frequent BMRs when the toroidal field is strong and vice versa. The BMR tilt has a Gaussian scatter around Joy's law with standard deviation $\left(\sigma_{\delta}\right)$ of $30^{\circ}\left(15^{\circ}\right)$ for Run B11 (B10). Another key ingredient of the model is the downward magnetic pumping which has the same form as in KM17 and it is given by

$$
\begin{gathered}
\gamma=-\frac{\gamma_{\mathrm{CZ}}}{2}\left[1+\operatorname{erf}\left(\frac{r-0.725 R_{\odot}}{0.01 R_{\odot}}\right)\right] \hat{\boldsymbol{r}} \\
-\frac{\left(\gamma_{\mathrm{S}}-\gamma_{\mathrm{CZ}}\right)}{2}\left[1+\operatorname{erf}\left(\frac{r-0.9 R_{\odot}}{0.02 R_{\odot}}\right)\right] \hat{\boldsymbol{r}}
\end{gathered}
$$

Table 1

Summary of simulations. Parameters of Runs B10-B11 are the same as in KM17, while for Run $2^{*} \Phi_{0}$ and $\sigma_{\delta}$ are different than the ones in KM17. Run B2* failed to recover after it entered into a grand minimum. $T_{\mathrm{sim}}$, and $f_{\mathrm{GM}}$ denote the length of simulation, and $\%$ of time spent in grand minima (GM), respectively.

\begin{tabular}{ccccccc}
\hline Run & $\Phi_{0}$ & $\gamma_{\mathrm{CZ}}, \gamma_{\mathrm{S}}\left(\mathrm{m} \mathrm{s}^{-1}\right)$ & $\sigma_{\delta}$ & $T_{\mathrm{sim}}(\mathrm{yr})$ & \# of GM & $f_{\mathrm{GM}}$ \\
\hline B10 & 2.4 & 2,20 & $15^{\circ}$ & 11650 & 17 & $11 \%$ \\
B11 & 2.4 & 2,20 & $30^{\circ}$ & 19214 & 46 & $17 \%$ \\
$\mathrm{~B}^{*}$ & 65 & 0,0 & $30^{\circ}$ & 589 & 1 & $\ldots$ \\
B14 & 2.4 & 2,22 & $30^{\circ}$ & 2952 & 5 & $17 \%$ \\
\hline
\end{tabular}

Note. - The parameter $\Phi_{0}$ is used to boost the observed flux distribution in our model; see Equation 8 of KM17 for details.

where $\gamma_{\mathrm{CZ}}=2 \mathrm{~m} \mathrm{~s}^{-1}$ and $\gamma_{\mathrm{S}}=20 \mathrm{~m} \mathrm{~s}^{-1}$. Thus, the pumping $\gamma$ has a value of $\gamma_{S}$ only near the surface, while in the rest of the $\mathrm{CZ}$ it is $\gamma_{\mathrm{CZ}}$.

Pumping is a process in which the magnetic flux can be transported in a stratified convective medium due to the topological asymmetry in the convective flow. A variety of theoretical and numerical models suggest that it is operating in the solar CZ, particularly near the surface where both the velocity amplitude and the density stratification are largest (e.g., Drobyshevski \& Yuferev 1974; Krause \& Rädler 1980; Tobias et al. 1998, Petrovay \& Szakaly 1993). Although at present, we do not have a stringent constraint on the strength of the pumping, previous studies (Käpylä et al. 2006, Karak et al. 2014b) suggest its value to be at least a few tenths of the convective velocity. Keeping in mind that the upper layer of the Sun is highly convective with the observed surface convection speed of a few $\mathrm{km} \mathrm{s}^{-1}$, a value of $20 \mathrm{~m} \mathrm{~s}^{-1}$ pumping speed is realistic (Spruit 1997; Nordlund et al. 2009). This value is also large enough to make the magnetic field near the surface approximately vertical, which improves the agreement between Babcock-Leighton dynamo models and Surface Flux Transport (SFT) models ${ }^{1}$.

\section{RESULTS AND DISCUSSION}

A time series of sunspot number (SSN) obtained from Run B11 is shown in Figure 1(a); see Table 1 for parameters and KM17 for details of how the sunspots are produced in this model based on the toroidal field in the interior. We note that this SSN is smoothed using the same procedure as done in Usoskin et al. (2007), that is, we first bin the monthly SSN in 10-year intervals and then filter the data by averaging over five neighboring points using the Gleissberg's lowpass filter 1-2-2-2-1 (Gleissberg 1944). The blue shading areas indicate the grand minima which are defined when the smoothed SSN goes below $50 \%$ of the mean for at least two consecutive decades (the same procedure as given in Usoskin et al. (2007)). To display the variation of the original SSN, including its 11-year periodicity, we show the monthly SSN variation for about 1600 years in Figure 1. b). In Figure 1)(a),

1 Cameron et al. (2012) showed that the inclusion of magnetic pumping in Babcock-Leighton dynamo models can improve their agreement with SFT models. The radially downward magnetic pumping makes the field more radial near the surface and suppresses the vertical diffusion of the magnetic flux. To do this the radial advection of the field through downward pumping has to dominate over the diffusion. They showed that this requires $5 l / \gamma_{\mathrm{S}}=$ $l^{2} / \eta$, where $l=0.1 R_{\odot}$ the depth of the near-surface layer where pumping is operating, and $\eta$ is diffusion coefficient in the near-surface layer. Taking $\eta=$ $3 \times 10^{12} \mathrm{~cm}^{2} \mathrm{~s}^{-1}$, this gives the pumping speed $\gamma_{\mathrm{S}}=21.5 \mathrm{~m} \mathrm{~s}^{-1}$. Thus the value of $\gamma_{\mathrm{S}}$ used in our simulations is in agreement with this argument. 

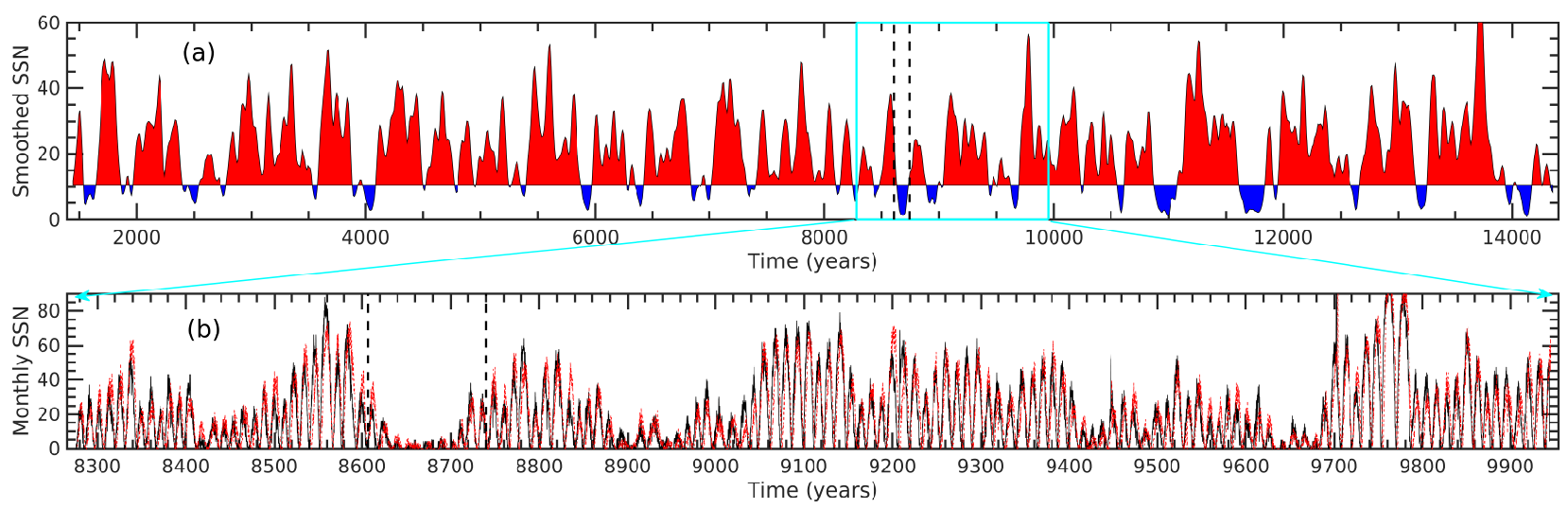

Figure 1. (a) Temporal variation of the smoothed SSN from 13,000-year simulation of Run B11. Blue shaded regions below the horizontal line represent the grand minima. (b) Monthly smoothed (with boxcar average of 3-month width) SSN (black/red: north/south) shown only for a selected 1600-year interval. Vertical dashed lines indicate the time-window which is focused in Figure 2

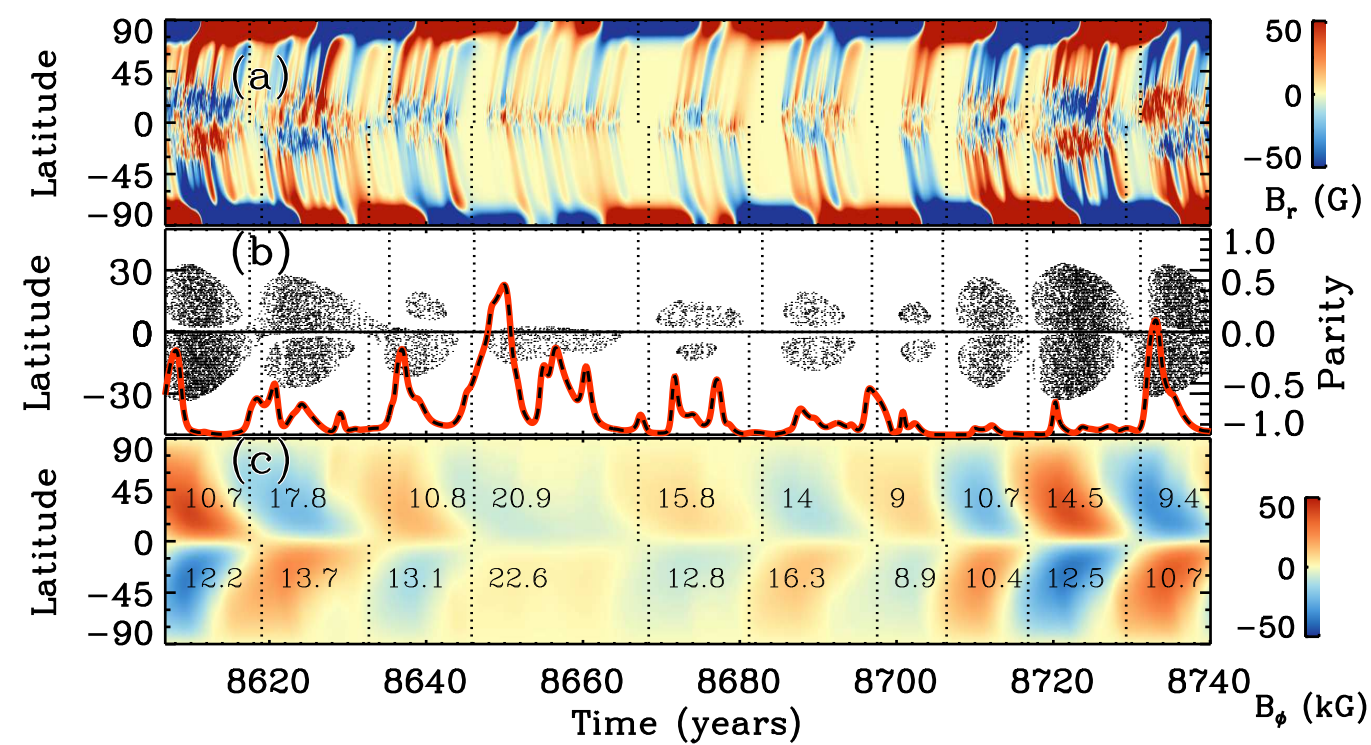

Figure 2. Zoomed-in view of a grand minimum from Figure 1 Latitude-time variations of (a) surface radial field, (b) sunspots, and (c) toroidal field at the BCZ. The red/dashed line in (b) shows the parity of the toroidal field at the BCZ (computed by cross-correlating the field between two hemispheres using Eq. 11 of KM17). We note that for perfect anti-symmetric (symmetric) toroidal field, the parity is expected to be -1 (1). Dotted lines mark the minima of sunspot cycles and periods in year are labeled in (c). The extrema of $B_{r}$ and $B_{\phi}$ are $[-1561,1645] \mathrm{G}$ and $[-38,40] \mathrm{kG}$, respectively.

we notice several grand minima; see Run B11 in Table 1 . Durations of some of these grand minima are similar to the Maunder minimum and some are even longer.

To characterize the features of the grand minima produced in our model, we highlight a few cycles from 8615-8740 years in Figure 2 We notice that the period of the first few cycles during this grand minimum is slightly longer than the average period of 10.8 years. This is consistent with the solar activity during Maunder minimum obtained from ${ }^{14} \mathrm{C}$ data (Miyahara et al. 2004), although ${ }^{10} \mathrm{Be}$ data gives somewhat different picture (Fligge et al. 1999, Beer et al. 1998). The longer cycle period is expected when there are fewer BMRs at the beginning of the grand minimum because with few BMRs the new poloidal flux needs more time to accumulate and thus reverse the old polar flux. We further note that during grand minima, BMRs appear near the equator which is consistent with the observational findings during the Maunder minimum (Ribes \& Nesme-Ribes 1993). Low-latitude BMRs appearance in our model is a consequence of chosen latitude- dependent threshold field strength for BMR production; see KM17 for details. Another distinct feature of grand minima is the hemispheric asymmetry. Around the year 8660 in Figure 2(b), most BMRs are produced in the southern hemisphere (also see the parity of the field which is linked to the hemispheric asymmetry; KM17). A strong hemispheric asymmetry was also observed during the Maunder minimum (Ribes \& Nesme-Ribes 1993). All these features (longer period, BMRs emergence near equator, and hemispheric asymmetry) are not limited to this grand minimum shown in Figure 2, they are also observed in other grand minima.

In any Babcock-Leighton dynamo model, the only source of poloidal field is the tilted BMRs. Thus, BMR emergence is essential to enable our model to emerge from grand minima. In our model, the SSN during grand minima goes to a very small value but never becomes zero for much more than a year. For example, the mean spot number during years 8650 8710 in Figure 1 (b) is about 5.8, which is only $13 \%$ of the mean spot number from the entire simulation run. Thus the 


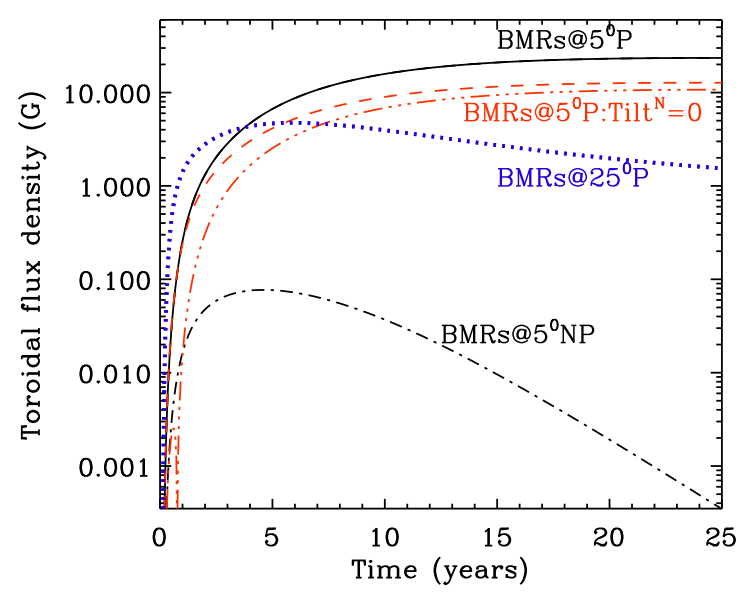

Figure 3. Evolution of the absolute value of the toroidal flux density obtained by averaging from $0^{\circ}$ to $30^{\circ}$ latitude at BCZ. The solid and dotted lines represent cases in which BMRs are deposited symmetrically at $\pm 5^{\circ}$ (BMRs@ @ $5^{\circ} \mathrm{P}$ ) and $\pm 25^{\circ}$ latitudes (BMRs@25 $@ 2$ ), respectively. The red lines (dash-double dotted for north and dashed for south) represent the case in which BMRs are deposited at $\pm 5^{\circ}$ but the northern BMR has zero tilt (BMRs@ $5^{\circ} \mathrm{P}: \mathrm{Tilt}^{\mathrm{N}}=0$ ). The dash-dotted line represents the case in which BMRs are deposited at $\pm 5^{\circ}$ but the pumping is set to zero (BMRs@ $5^{\circ} \mathrm{NP}$ ).

question is how those fewer sunspots during grand minima are capable of producing enough poloidal flux to maintain the dynamo?

It turns out that it is the downward magnetic pumping which enables our model to recover from grand minima even with a few sunspots. The magnetic pumping near the surface makes the poloidal field radial and suppresses the diffusion of the horizontal field through the surface, as shown by Cameron et al. (2012); Karak \& Cameron (2016). Thus when a few sunspots during grand minima produce poloidal flux, it remains in the CZ for many years. This poloidal flux continuously produces toroidal flux through the $\Omega$ effect. The pumping also does not allow this toroidal flux to diffuse through the solar surface. (The toroidal flux can diffuse across the equator but this diffusion can be balanced by its generation.)

To validate this idea, we examine the magnetic field generated from the decay of two BMRs in this model. We perform a simulation by depositing one BMR at $5^{\circ}$ latitude and another at $-5^{\circ}$ latitude as an initial condition, with no other seed field present. Tilts of these BMRs are given by Joy's law with no scatter around it. The flux and other properties of these BMRs are identical. The polar flux produced from the decay of these BMRs eventually produces toroidal flux near the BCZ as shown by the solid line (case: BMRs@ $5^{\circ} \mathrm{P}$ ) in Figure 3. Now we repeat the same experiment by switching off the magnetic pumping. The dash-dotted line in Figure 3 represents this simulation (case: BMRs@ $5^{\circ} \mathrm{NP}$ ). We find that without pumping the toroidal flux becomes orders of magnitude smaller and decays indefinitely. Within the context of grand minima, this implies that the poloidal flux produced by even a few BMRs will remain in the $\mathrm{CZ}$ long enough to be converted to toroidal flux through the differential rotation. Eventually, the toroidal flux will become strong enough to trigger more BMR emergence, bringing the model out of the grand minimum.

We note that the recent $2 \mathrm{D} \times 2 \mathrm{D}$ model of $\mathrm{LC} 17$, which uses a much smaller tilt scatter than we have used in the present simulation, shuts off entirely whenever it enters into a Maunder-like extended minimum. While there are many fun- damental differences between their model and ours, the major difference is that their model does not take into account magnetic pumping. In their model, when SSN falls below a certain level for a few years, the toroidal field decays rapidly and once it falls below the threshold for the spot production, no new spot can form. This makes the dynamo shut off completely.

To probe the above conclusion even further, we repeat our grand minima simulation without magnetic pumping (Run $\left.\mathrm{B} 2^{*}\right)$. The tilt scatter and other parameters are the same as in Run B11. However, the spot flux (parameter $\Phi_{0}$ in the model) is increased to 65 from 2.4. This change is needed in order to make the dynamo supercritical since pumping enhances the dynamo efficiency (Karak \& Cameron 2016). The output of this simulation is shown in Figure 4(c). As expected, when the magnetic pumping is not included, the model cannot recover from the grand minimum once it enters into it. Interestingly, when we restart this simulation with the snapshot right before it entered into the grand minimum ( $t=1750$ years) as the initial condition but with magnetic pumping, then it recovers.

Another feature of our model that is beneficial for recovery from grand minima is the spontaneous emergence of BMRs at low latitudes, as evident in Figure 2 b). These low-latitude spots are very efficient in generating poloidal flux in comparison to the higher latitude spots. To demonstrate that this is true, we repeat the same simulation of two symmetric BMRs as shown by the solid line in Figure 3(BMRs@ $\left.5^{\circ} \mathrm{P}\right)$, but instead of depositing them at $\pm 5^{\circ}$ latitudes, we deposit them at $\pm 25^{\circ}$ latitudes. As usual, the tilt is obtained from Joy's law. The dotted line in Figure 3 shows this simulation (note the $\log$ scale of the vertical axis); case: BMRs@ $25^{\circ} \mathrm{P}$. On comparing with solid line, we confirm that the BMR pair closer to the equator produces much larger toroidal flux, although they have smaller tilt. This is consistent with previous studies (Jiang et al. 2014, Hazra et al. 2017) which have shown that when BMR pairs emerge at low latitudes, the cancellation of flux across the equator is more efficient. Since this cancellation regulates the net flux in each hemisphere, it ultimately leads to stronger polar fields and, in turn, stronger toroidal fields. In our model (and also in observations) BMRs during grand minima are produced closer to the equator and these few low-latitude BMRs help the dynamo re-establish normal activity by enhancing the poloidal field generation.

Furthermore, our model has a strong hemispheric coupling (through the turbulent diffusion). Due to this, if one hemisphere (for example, the northern hemisphere around 8660 years in Figure 2(b)) does not get many BMRs, the other hemisphere can supply some poloidal flux. Thus strong hemispheric coupling is also beneficial for the dynamo to recover from grand minima.

The relatively large tilt scatter in our model $\left(\sigma_{\delta}=30^{\circ}\right)$ can have a particularly important influence during grand minima, when the number of BMRs is small. However, if a BMR pair near the equator gets very different tilts than given by Joy's law, then a significant polar flux may be produced, unless when both BMRs have zero tilts or the same tilts with the same polarity (i.e., one Hale and other anti-Hale). For example, when one pair in one hemisphere has zero tilt and the other pair's tilt is given by Joy's law, then they still, produce a significant polar flux; see the red lines (dashdouble dot for north and dashed for south) in Figure 3 (case: BMRs@5 $5^{\circ}$ :Tilt ${ }^{\mathrm{N}}=0$ ).

We may ask the question, 'Can the tilt scatter ever be large 


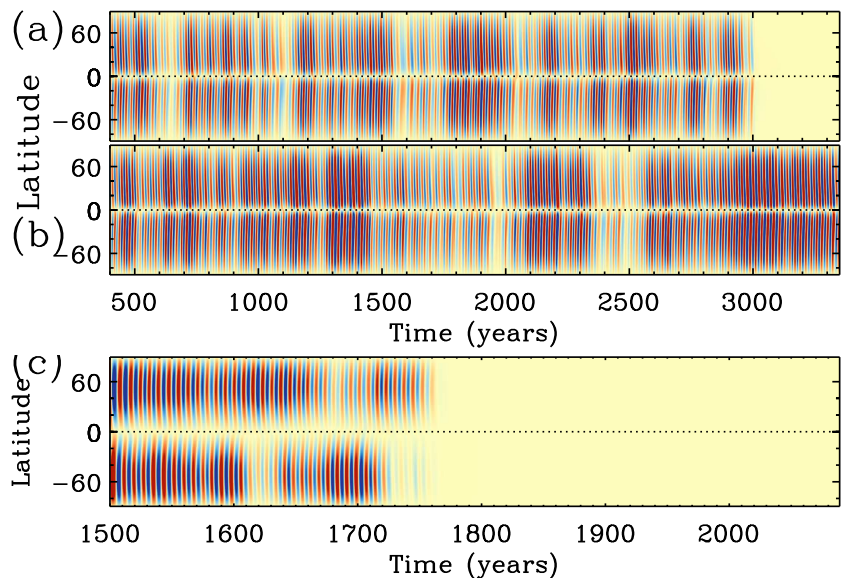

Figure 4. Latitude-time variation of the toroidal field at the BCZ. (a) Demonstrates a case out of total 19,200 years of simulations (Run B11) which could not recover from a grand minimum. (b) Obtained from Run B14 which is the same as (a) except higher pumping. (c) From Run B2* (without magnetic pumping).

enough to make the poloidal flux extremely weak and the toroidal flux remains below the threshold for several years to produce no new spot?' If this happens, then the dynamo may fail to recover from a grand minimum. To explore this, we initiate different realizations of Run B11 by using different random seeds for the tilt angle scatter, the time delay, and the BMR flux distribution. In about 19,200 years of total simulation time, we found a case in which the model failed to recover from a grand minimum and the dynamo shut off completely; see Figure 4(a). In this case, the model could not recover because SSN went to a very low value for many years and the poloidal field generated from those few spots could not overcome the diffusion of the fields. However, the most interesting fact is that when we repeat this simulation with the same initial condition and same realizations of fluctuations but with an increased magnetic pumping $\gamma_{\mathrm{S}}$ of $22 \mathrm{~m} \mathrm{~s}^{-1}$ (instead of $20 \mathrm{~m} \mathrm{~s}^{-1}$, which is the value for Runs B10-11), we do not get any dying dynamo; see Run B14 in Table 1 and Figure 4(b). This slight increase in the magnetic pumping is enough to enable the dynamo to recover from all grand minima as discussed above.

\section{CONCLUSION}

We find that the Babcock-Leighton process can still operate during grand minima even with a few spots. This result was unexpected and it strikingly contrasts to previous studies (Karak \& Choudhuri 2013, Hazra et al. 2014; Passos et al. 2014, Lemerle \& Charbonneau 2017), which suggested that the Babcock-Leighton process cannot operate during Maunder-like minima. The Babcock-Leighton dynamo, of course, cannot operate when there are no sunspots and that can happen if the magnetic field during grand minima goes to a very small value (below the threshold for spot generation). However, we expect that this is not happening in Sun because during grand minima, at least during the Maunder minimum, there were still some sunspots (Vaquero et al. 2016, Zolotova \& Ponyavin 2016).

We have demonstrated that magnetic pumping can sustain a Babcock-Leighton dynamo throughout a grand minimum and enable it to re-establish normal activity. It achieves this by suppressing diffusive losses, allowing toroidal magnetic flux to accumulate and amplify until it is large enough to produce sunspots (BMRs). The few sunspots during the grand mini- mum are enough to sustain the cycle, in part because they tend to emerge at low latitudes, which maximizes the efficiency of poloidal flux generation. In contrast to other BabcockLeighton models, there is no need to invoke an alternative source of poloidal field such as a turbulent $\alpha$-effect. The sporadic appearance of sunspots at low latitudes in the model, often with substantial north-south asymmetry, is reminiscent of sunspot observations during the Maunder Minimum. Our results therefore suggest that the Babcock-Leighton mechanism may have been sufficient to sustain the solar cycle throughout the Maunder Minimum and into its subsequent recovery, with similar implications for previous grand minima.

We thank the anonymous referee, Mausumi Dikpati, Ricky Egeland, and Lisa Upton for reading this manuscript and providing valuable comments. We also thank Robert Cameron and Dibyendu Nandi for past inspiring discussions related to this study. BBK is supported by the NASA Living With a Star Jack Eddy Postdoctoral Fellowship Program, administered by the University Corporation for Atmospheric Research. The National Center for Atmospheric Research is sponsored by the National Science Foundation. Computations were carried out with resources provided by NASA's High-End Computing program (Pleiades) and by NCAR (Yellowstone).

\section{REFERENCES}

Arlt, R., Senthamizh Pavai, V., Schmiel, C., \& Spada, F. 2016, A\&A, 595, A104

Babcock, H. W. 1961, ApJ, 133, 572

Beer, J., Blinov, A., Bonani, G., Hofmann, H. J., \& Finkel, R. C. 1990, Nature, 347, 164

Beer, J., Tobias, S., \& Weiss, N. 1998, Sol. Phys., 181, 237

Cameron, R. H., Schmitt, D., Jiang, J., \& Işık, E. 2012, A\&A, 542, A127

Charbonneau, P. 2010, Liv. Rev. Sol. Phys., 7, 3

Charbonneau, P., Blais-Laurier, G., \& St-Jean, C. 2004, ApJ, 616, L183

Choudhuri, A. R., \& Karak, B. B. 2009, Res. Astron. Astrophys., 9, 953

—. 2012, Phys. Rev. Lett., 109, 171103

Dasi-Espuig, M., Solanki, S. K., Krivova, N. A., Cameron, R., \& Peñuela, T. 2010, A\&A, 518, A7

Drobyshevski, E. M., \& Yuferev, V. S. 1974, Journal of Fluid Mechanics, 65,33

D’Silva, S., \& Choudhuri, A. R. 1993, A\&A, 272, 621

Eddy, J. A. 1976, Science, 192, 1189

Fligge, M., Solanki, S. K., \& Beer, J. 1999, A\&A, 346, 313

Gleissberg, W. 1944, Terrestrial Magnetism and Atmospheric Electricity

(Journal of Geophysical Research), 49, 243

Hazra, G., Choudhuri, A. R., \& Miesch, M. S. 2017, ApJ, 835, 39

Hazra, S., Passos, D., \& Nandy, D. 2014, ApJ, 789, 5

Inceoglu, F., Arlt, R., \& Rempel, M. 2017, ApJ, 848, 93

Jiang, J., Cameron, R. H., \& Schüssler, M. 2014, ApJ, 791, 5

-. 2015, ApJ, 808, L28

Käpylä, P. J., Korpi, M. J., Ossendrijver, M., \& Stix, M. 2006, A\&A, 455, 401

Karak, B. B., \& Cameron, R. 2016, ApJ, 832, 94

Karak, B. B., \& Choudhuri, A. R. 2013, Res. Astron. Astrophys., 13, 1339

Karak, B. B., Jiang, J., Miesch, M. S., Charbonneau, P., \& Choudhuri, A. R. 2014a, Space Sci. Rev., 186, 561

Karak, B. B., \& Miesch, M. 2017, ApJ, 847, 69

Karak, B. B., Rheinhardt, M., Brandenburg, A., Käpylä, P. J., \& Käpylä, M. J. 2014b, ApJ, 795, 16

Krause, F., \& Rädler, K. H. 1980, Mean-field magnetohydrodynamics and dynamo theory (Oxford: Pergamon Press)

Leighton, R. B. 1964, ApJ, 140, 1547

Lemerle, A., \& Charbonneau, P. 2017, ApJ, 834, 133

Miesch, M. S., \& Dikpati, M. 2014, ApJ, 785, L8

Miesch, M. S., \& Teweldebirhan, K. 2016, Space Sci. Rev.

Miyahara, H., Masuda, K., Muraki, Y., Furuzawa, H., Menjo, H., \&

Nakamura, T. 2004, Sol. Phys., 224, 317

Nordlund, Å., Stein, R. F., \& Asplund, M. 2009, Living Reviews in Solar Physics, 6, 2 
Olemskoy, S. V., \& Kitchatinov, L. L. 2013, ApJ, 777, 71

Passos, D., Nandy, D., Hazra, S., \& Lopes, I. 2014, A\&A, 563, A18

Petrovay, K., \& Szakaly, G. 1993, A\&A, 274, 543

Ribes, J. C., \& Nesme-Ribes, E. 1993, A\&A, 276, 549

Solanki, S. K., Usoskin, I. G., Kromer, B., Schüssler, M., \& Beer, J. 2004, Nature, 431, 1084

Spruit, H. 1997, MmSAI, 68, 397

Stenflo, J. O., \& Kosovichev, A. G. 2012, ApJ, 745, 129
Tobias, S. M., Brummell, N. H., Clune, T. L., \& Toomre, J. 1998, ApJ, 502, L177

Usoskin, I. G., Solanki, S. K., \& Kovaltsov, G. A. 2007, A\&A, 471, 301 Vaquero, J. M., et al. 2016, Sol. Phys., 291, 3061

Wang, Y.-M., Colaninno, R. C., Baranyi, T., \& Li, J. 2015, ApJ, 798, 50

Zolotova, N. V., \& Ponyavin, D. I. 2016, Sol. Phys., 291, 2869 